\title{
Residual-limb quality and functional mobility 1 year after transtibial amputation caused by vascular insufficiency
}

\author{
Henk J. Arwert, MD; ${ }^{*}$ Mirjam H. van Doorn-Loogman, MD; ${ }^{2}$ Jan Koning, PhD, MD; ${ }^{3}$ Martinus Terburg, MD; ${ }^{3}$ \\ Mathilde Rol, MD; ${ }^{2}$ Marij E. Roebroeck, $\mathbf{P h D}^{\mathbf{4}}$ \\ Sophia Rehabilitation Center, ${ }^{1}$ Outpatient Clinic, Westeinde Hospital and ${ }^{2}$ Clinical Rehabilitation, The Hague, the Neth- \\ erlands; ${ }^{3}$ Reinier de Graaf Group, Delft, the Netherlands; ${ }^{4}$ Erasmus Medical Center, Rotterdam, the Netherlands
}

\begin{abstract}
This study identified which residual-limb quality factors are related to functional mobility 1 year after transtibial (TT) amputation. A group of 28 TT amputees were evaluated with respect to their functional mobility (Prosthesis Evaluation Questionnaire [PEQ], Locomotor Index, Timed Up and Go test). The general (Chakrabarty score) and bony (tibial length, relative fibular length) residual-limb quality factors were assessed. An increase in general residual-limb quality (Chakrabarty $>60$ ) was correlated with greater functional mobility in one of the outcome measures (PEQ). For bony residual-limb quality, a tibial length of $12-15 \mathrm{~cm}$ distal from the knee joint line was correlated with greater functional outcome for all three outcome measures and the relative fibular length was not correlated with functional mobility for any of the outcome measures. This study showed that specific aspects of residuallimb quality are related to increased functional mobility. The amputation technique and resulting residual-limb factors may be important for patients to achieve functional prosthetic use.
\end{abstract}

Key words: fibula, functional mobility, Locomotor Index, Prosthesis Evaluation Questionnaire, prosthetics, rehabilitation, residual limb, tibia, Timed Up and Go test, transtibial amputation.

\section{INTRODUCTION}

Transtibial (TT) amputation is a surgical procedure that has been performed for centuries. Originally, it was a lifesaving procedure on the battlefield. Later it became the end point of surgical treatment for a vascular patient or, from another perspective, the starting point of a rehabilitation treatment aimed at restoring mobility with a prosthesis [1-2].

Despite this long history, the systematic evaluation of the amputation technique has only become a point of interest in the last decade [3-5]. We hypothesized that residual-limb quality may be important for the effectiveness of rehabilitation.

No consensus in the literature exists with respect to tibial length; a range of measures have been given, including "one hand's breadth" [6], no advice at all [7], $20 \mathrm{~cm}$ [8], 9-11 cm distal from the knee joint line as ideal for the ischemic patient [9], $8 \mathrm{~cm}$ distal to the tibial tuberosity [10], and 4-7 in. [11]. We also noted a lack of consensus for other residual-limb factors, such as the length of the fibula. The question still remains: What are the characteristics of an adequate residual limb? The International Society for Prosthetics and Orthotics (ISPO) has devised a clinical standard for measurement and classification of the residual limb [12]. Based on this standard,

\footnotetext{
Abbreviations: ISPO $=$ International Society for Prosthetics and Orthotics, $\mathrm{PEQ}=$ Prosthesis Evaluation Questionnaire, $\mathrm{TT}=$ transtibial, TUGT $=$ Timed Up and Go test.

* Address all correspondence to Henk J. Arwert, MD; Sophia Rehabilitation Center, Outpatient Clinic, Westeinde Hospital, PO Box 432, 2501 CK The Hague, the Netherlands; +31-703303758; fax: +31-703303242.

Email: hjarwert@knmg.nl
}

DOI: 10.1682/JRRD.2006.05.0047 
Chakrabarty developed a scoring system with a useful format for the clinical setting. This scoring system covers several aspects of residual-limb quality, including wound healing and soft tissue condition.

This study investigated whether residual-limb quality was related to functional mobility 1 year after amputation. In addition to the soft tissue conditions, we specifically focused on evaluating the bony aspects of the residual limb because these can be adequately assessed 1 year after amputation. A better insight into these aspects of residuallimb quality may inform optimal surgical procedures.

\section{MATERIALS AND METHODS}

\section{Patients}

In this study, we focused on patients with a unilateral TT amputation caused by vascular insufficiency. Patients were evaluated 1 year after a TT amputation. Patients were recruited from five hospitals in the southwest region of the Netherlands by means of operating room records. We evaluated a retrospective cohort of 117 patients that underwent a TT amputation. Inclusion criteria were (1) $\geq 18$ years, (2) unilateral TT amputation caused by vascular insufficiency, and (3) ability to visit the outpatient clinic.

In total, 71 patients met the inclusion criteria. A relevant number of patients $(n=28)$ died, as could be expected [13-15]. Four patients could not be traced because of incorrect addresses. Of the 39 patients contacted, 28 were willing to participate in the study, a 72 percent response rate. Of the 28 subjects who participated in the study, 5 were unable to use their prosthesis at the time of data collection because of an ulcer, pain, or other problems. We were unable to collect certain data on these subjects, and therefore, they were not included in the statistical analysis.

All participants gave written informed consent. The research protocol was approved by the Medical Ethics Committee of Leiden University Medical Center.

\section{Determinants}

We assessed general residual-limb quality with the scoring system developed by Chakrabarty [5] (Appendix, available online only at http://www.rehab.research.va.gov/). The Chakrabarty score is a graded summed score that includes soft tissue condition, wound healing, and joint mobility. The maximum possible score is 100 points. Chakrabarty indicated a score of $\geq 60$ points as a high- quality residual limb (grade A) and a score of $<60$ points as a low-quality residual limb (grade B). This system is based on the clinical standard for measurement and classification of residual limbs devised by the ISPO [12]. Face validity of one aspect of the scoring system, i.e., wound healing, was confirmed by a survey of 66 experts involved in surgery and rehabilitation of amputees and a literature review [5].

We also assessed whether the bony aspects of the residual limb were adequate: the tibial length and the relative fibular length were measured by radiographs. We positioned a calibrated radiopaque grid next to the tibia to correct for scaling factors introduced by the radiographic technique. All measurements were carried out by two independent observers (Drs. van Doorn-Loogman and Terburg). In the case of different outcomes, radiographs were reevaluated until consensus was obtained.

Prior to initiation of the study, we determined the following criteria, based on expert opinion, for distinguishing a high- from a low-quality residual limb: the tibial length should be 12-15 cm distal from the knee joint line and the fibular length should be $2-3 \mathrm{~cm}$ shorter than the tibial length. We were interested in using the quality of the residual limb to determine functional outcome. We expected increased functional mobility after 1 year in the subgroups with a tibial length of $12-15 \mathrm{~cm}$ and a fibular length $2-3 \mathrm{~cm}$ shorter than the tibia.

\section{Outcome Measures}

The functional use of a prosthesis was evaluated with the ambulation subscale of the Prosthesis Evaluation Questionnaire (PEQ) [16], the Locomotor Index [17] and the Timed Up and Go test (TUGT) [18-19].

The ambulation subscale of the PEQ consists of eight items on the level of prosthetic use and comfort during walking, stair climbing, and walking on uneven ground that are assessed by means of a visual analogue scale. Mean scores for all questions were included in the analysis.

The Locomotor Index is part of the Prosthetic Profile of the Amputee. It assesses 14 activities, including rising from a chair, walking, picking up an object from the floor, and stair-climbing and whether a person performs these activities independently or needs help from another person. Answers are graded on a 4-point scale, from 0 (not able) to 3 (performing independently). The maximum possible score is 42 points. The Locomotor Index has been validated in a mixed group of prosthesis users.

The TUGT is a validated walking test. The subject is asked to stand up from a seated position, walk $3 \mathrm{~m}$, turn 
around, walk back, and sit again. The time in seconds is recorded. Thus, a shorter time reflects a better score.

\section{Risk Factors}

We accounted for the following variables: age, sex, diabetes mellitus, comorbidity (categorized into four comorbidity groups: cardiopulmonary, neurological, orthopedic, and optical), and ambulation level 1-month preoperatively (assessed retrospectively by means of an interview, walking distance of $<100 \mathrm{~m}$ was considered poor).

\section{Data Analyses}

Patients were categorized into subgroups by means of the summed Chakrabarty scores [5]; those having a score $\geq 60$ points (grade A) and those having a score $<60$ points (grade B). We performed chi-square analysis to determine whether significant differences in risk factors existed between subgroups with higher and lower residual-limb quality.

The bivariate correlations between residual-limb quality and adequate bony lengths and functional outcome were assessed with the Kendall $\tau$ rank correlation coefficient (ordinal level). Because of the relatively small sample size, we estimated partial correlations (Pearson $r$ ) to investigate whether relationships between residuallimb quality factors and functional mobility outcomes would diminish when risk factors (age, sex, diabetes mellitus, comorbidity, or preoperative ambulation level) were controlled for individually [20].

Differences in functional mobility outcomes between subgroups with higher and lower residual-limb quality and with adequate and inadequate tibial length and relative fibular length were tested with a $t$-test for independent samples ( $\alpha \leq 0.05)$.

\section{RESULTS}

Descriptive statistics of the patients are given in Table 1. Of the 23 patients with a prosthesis, 12 had a grade A residual limb according to Chakrabarty's score, 11 had a tibial length of $12-15 \mathrm{~cm}$, and 12 had a fibular length 2-3 cm shorter than the tibia. No subjects had a tibial length longer than $15 \mathrm{~cm}$. In our study, the distribution of participants by age, sex, and prevalence of diabetes mellitus was similar to that in other studies [13-15].

Of the 23 patients with a prosthesis, 2 did not complete the PEQ adequately and 5 did not complete the Locomotor Index; 4 patients were unable to perform the TUGT. The radiographs of one patient were missing.

The distribution of risk factors in the dichotomized subgroups is given in Table 2. The risk factors were evenly distributed over the subgroups with three exceptions: one subgroup (Chakrabarty $\geq 60$ ) included more subjects with diabetes mellitus, another subgroup (tibial

Table 1.

Descriptive statistics for patients who did $(n=23)$ or did not $(n=5)$ use a prosthesis 1 year postamputation. Data presented as number of subjects unless otherwise noted.

\begin{tabular}{lcc}
\hline \multicolumn{1}{c}{ Variable } & Prosthesis & No Prosthesis \\
\hline Age (mean \pm SD) & $69.8 \pm 11.1$ & $63.6 \pm 10.3$ \\
Sex (male/female) & $15 / 8$ & $2 / 3$ \\
Diabetes Mellitus & 14 & 3 \\
$\geq 2$ Comorbidities & 12 & 5 \\
Preop Walk $<100 \mathrm{~m}$ & 15 & 3 \\
Chakrabarty $\geq 60$ & 12 & 1 \\
Tibial Length 12-15 cm & 11 & 3 \\
Fibular Length 2-3 cm Shorter & 12 & 2 \\
\hline \multicolumn{2}{l}{ Preop = preoperatively, SD = standard deviation. } \\
\hline
\end{tabular}

Table 2.

Distribution of risk factors in patients with prosthesis $(n=23)$ specified for subgroups of patients with high or low general (Chakrabarty score) or bony (tibial length and relative fibular length) residual-limb quality. Cells with a gray background indicate significant difference between subgroups ( $p<0.05$, chi-square test). Data presented as number of subjects unless otherwise noted.

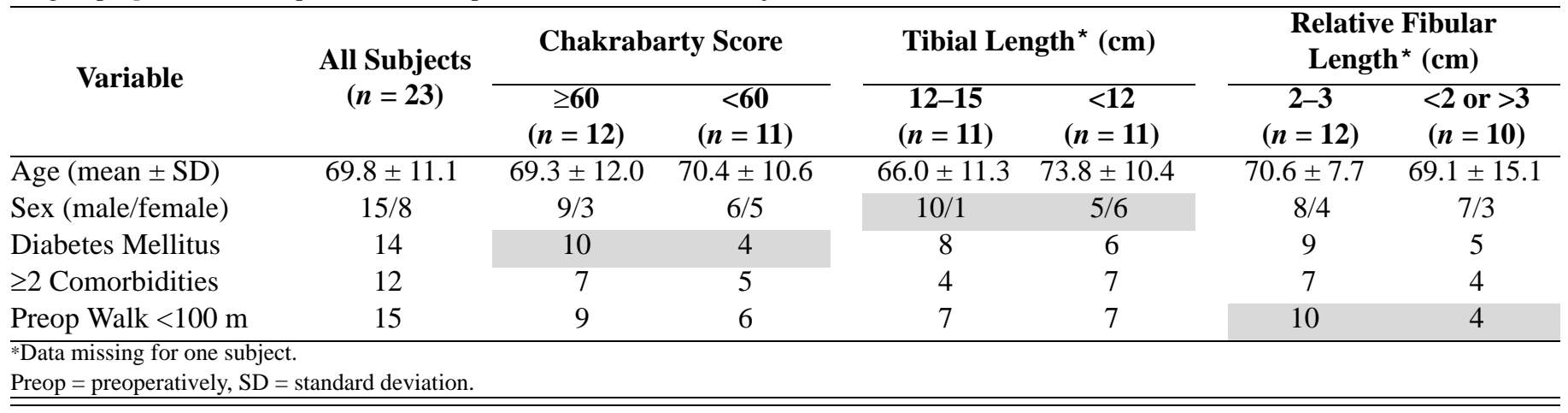


length $12-15 \mathrm{~cm}$ ) included more males, and one subgroup (fibular length $2-3 \mathrm{~cm}$ shorter) included more subjects with decreased preoperative walking ability ( $p<$ 0.05).

The relationships between the determinants and the outcome measures are summarized in the Figure. The patients with a higher residual-limb quality, i.e., Chakrabarty $\geq 60$, scored significantly higher on the PEQ ( $n=$ 21, $p=0.001$, Kendall $\tau=0.51$ ) and trended toward better performance on the TUGT ( $n=19, p=0.085$, Kendall $\tau=-0.46)$.

Results for the bony aspects of the residual limb showed that the tibial length was of significant influence in all three outcome measures, with a tibial length of 12 $15 \mathrm{~cm}$ leading to better scores than a tibial length $<12 \mathrm{~cm}$. This result was true for the PEQ $(n=21, p=$ 0.001 , Kendall $\tau=0.58)$, the Locomotor Index $(n=18$, $p=0.005$, Kendall $\tau=0.68$ ), and the TUGT ( $n=19, p=$ 0.029 , Kendall $\tau=-0.57$ ). The relative fibular length was not associated with functional mobility on any of the three outcome measures. For all determinants, similar correlations with outcome measures were found after controlling for every risk factor.

\section{DISCUSSION}

Because the demographic data of our patient group was similar to that in other studies and considering the acceptable response rate of 72 percent, our sample of dysvascular TT amputees may be considered representative.

We analyzed the distribution of the risk factors (i.e., age, sex, diabetes mellitus, preoperative ambulation level, and comorbidity) because of the retrospective nature of this study. We found no significant differences between the subgroups of patients with high or low residual-limb quality for 12 of the 15 dichotomized subgroups. In addition, correction for risk factors and other residual-limb characteristics did not change the main results. Therefore, bivariate correlations between determinants of residual-limb quality and functional outcome were analyzed; the number of subjects in each subgroup was sufficient.

We considered the subjects without a prosthesis $(n=$ 5) to have decreased functional mobility. Their level of comorbidity was higher compared with subjects who had a prosthesis. The bony residual-limb characteristics of the subjects without a prosthesis were comparable to the other subjects, but their Chakrabarty scores were lower;
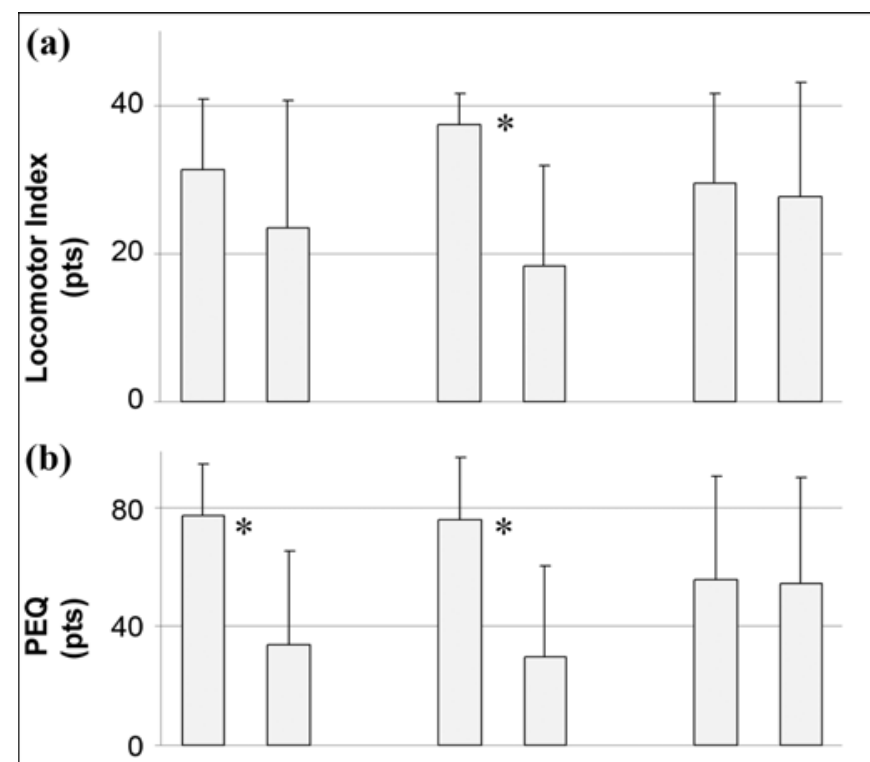

(c)

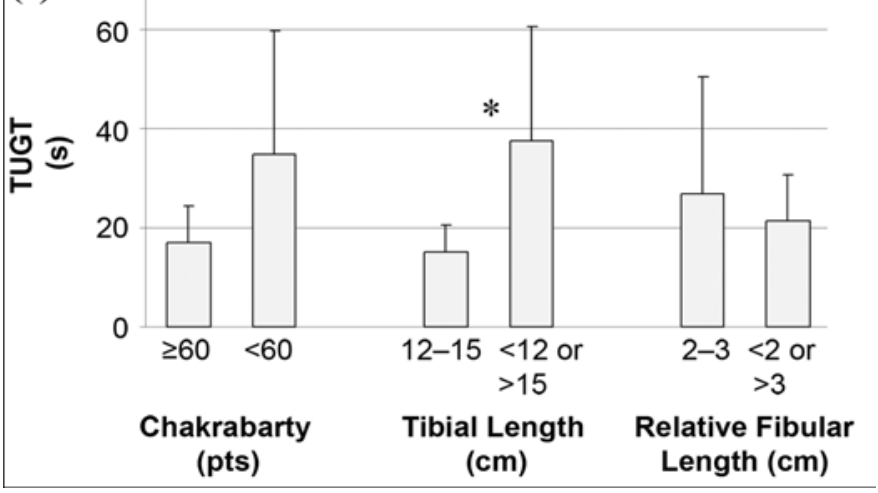

Figure.

Relationship between determinants (Chakrabarty score, tibial length, relative fibular length) and (a) Locomotor Index; (b) Prosthesis Evaluation Questionnaire (PEQ), ambulation subscale; and (c) Timed Up and Go test (TUGT). * indicates $p<0.05$ and error bars indicate +1 standard deviation. $\mathrm{pts}=$ points.

only one had a score $\geq 60$ points. The outcome measures used in this study focused primarily on the ability to walk with a prosthesis. Patients without a prosthesis were not included in the analyses.

Of the 23 patients with a prosthesis, 12 had an adequate residual limb, according to Chakrabarty's score. We found that patients with higher quality residual limbs (Chakrabarty $\geq 60$ ) had better outcomes [5] and, therefore, achieved the predicted mobility grade in less time. The relationship between residual-limb quality and mobility level was confirmed in our study. All three outcome measures trended in the same direction, and the relationship with the PEQ was statistically significant. The results 
demonstrate that residual-limb quality in general (skin quality, scars, shape, joint contracture, etc.) is related to achieved mobility 1 year postamputation. These findings underline the need for a meticulous surgical technique.

We expected that bony aspects of the residual limb would be important for functional mobility and this expectation was confirmed for tibial length. The PEQ, TUGT, and Locomotor Index were significantly related to tibial length. A tibial length of $12-15 \mathrm{~cm}$ distal from the knee joint line was related to a better outcome than a tibial length shorter than $12 \mathrm{~cm}$. Because we had no subjects with tibial lengths longer than $15 \mathrm{~cm}$, we cannot draw a conclusion about this specific subgroup.

The relative fibular length was not a conclusive factor, and no significant effect may exist as long as the fibula is shorter than the tibia.

\section{CONCLUSIONS}

Functional use of a prosthesis 1 year after TT amputation is related to residual-limb quality. This result is true for residual-limb quality in general as well as for specific bony aspects of the residual limb. In this study, a tibial length of 12-15 cm from the knee joint line correlated with a better functional outcome than shorter tibial lengths. The results regarding the relative fibular length are inconclusive. These findings underline the need for a meticulous surgical technique and attention to the residuallimb length that ensures good functional outcome after TT amputation.

\section{ACKNOWLEDGMENTS}

This material is the result of work supported with resources and the use of facilities at the Sophia Rehabilitation Center, Outpatient Clinic, The Hague, the Netherlands.

The authors have declared that no competing interests exist.

\section{REFERENCES}

1. Turney BW, Kent SJ, Walker RT, Loftus IM. Amputations: No longer the end of the road. J R Coll Surg Edinb. 2001; 46(5):271-73. [PMID: 11697693]

2. Taylor SM, Kalbaugh CA, Blackhurst DW, Hamontree SE, Cull DL, Messich HS, Robertson RT, Langan EM, York JW,
Carsten CG, Snyder BA, Jackson MR, Youkey JR. Preoperative clinical factors predict postoperative functional outcomes after major lower limb amputation: An analysis of 553 consecutive patients. J Vasc Surg. 2005;42(2):227-35. [PMID: 16102618]

3. Silver-Thorn MB, Childress DS. Parametric analysis using the finite element method to investigate prosthetic interface stresses for persons with trans-tibial amputation. J Rehabil Res Dev. 1996;33(3):227-38. [PMID: 8823671]

4. Cosgrove CM, Thornberry DJ, Wilkins DC, Ashley S. Surgical experience and supervision may influence the quality of lower limb amputation. Ann R Coll Surg Engl. 2002; 84(5):344-47. [PMID: 12398130]

5. Chakrabarty BK. An audit of the quality of the stump and its relation to rehabilitation in lower limb amputees. Prosthet Orthot Int. 1998;22(2):136-46. [PMID: 9747998]

6. Allcock PA, Jain AS. Revisiting transtibial amputation with the long posterior flap. Br J Surg. 2001;88(5):683-86. [PMID: 11350441$]$

7. Smith DG, Fergason JR. Transtibial amputations. Clin Orthop. 1999;(361):108-15. [PMID: 10212603]

8. Zollinger R. Atlas of surgical procedures. Philadelphia (PA): McGraw-Hill; 1992.

9. Morris PJ, Malt RA. Oxford textbook of surgery. New York (NY): Oxford University Press; 1994.

10. Rutherford RB. Vascular surgery. Philadelphia (PA): W.B. Saunders Company; 1977.

11. Burgess EM, Romano RL, Zettl JH. The management of lower extremity amputations. Chapter 2 . Washington (DC): U.S. Government Printing Office; 1969.

12. Persson BM, Liedberg E. A clinical standard of stump measurement and classification in lower limb amputees. Prosthet Orthot Int. 1983;7(1):17-24. [PMID: 6856447]

13. Pinzur MS, Gottschalk F, Smith D, Shanfield S, De Andrade R, Osterman H, Roberts J, Orlando-Crombleholme P, Larsen J, Rappazzini P, Bockelman P. Functional outcome of below-knee amputation in peripheral vascular insufficiency: A multicenter study. Clin Orthop Relat Res. 1993;(286):247-49. [PMID: 8425353]

14. Uiterwijk AE, Remerie SC, Rol M, Sier JC, Stam HJ, Terburg M. Routing through the health care system and level of functioning of lower limb amputees. Clin Rehabil. 1997; 11(3):253-62. [PMID: 9360039$]$

15. Rommers GM, Vos LD, Groothoff JW, Schuiling CH, Eisma WH. Epidemiology of lower limb amputees in the north of the Netherlands: Aetiology, discharge destination and prosthetic use. Prosthet Orthot Int. 1997;21(2):92-99. [PMID: 9285952]

16. Legro MW, Reiber GD, Smith DG, Del Aguila M, Larsen J, Boone D. Prosthesis Evaluation Questionnaire for persons with lower limb amputations: Assessing prosthesis-related quality of life. Arch Phys Med Rehabil. 1998;79(8):931-38. [PMID: 9710165] 
JRRD, Volume 44, Number 5, 2007

17. Treweek SP, Condie ME. Three measures of functional outcome for lower limb amputees: A retrospective review. Prosthet Orthot Int. 1998;22(3):178-85. [PMID: 9881605]

18. Mathias S, Nayak US, Isaacs B. Balance in elderly patients: The "get-up and go" test. Arch Phys Med Rehabil. 1986; 67(6):387-89. [PMID: 3487300]

19. Schoppen T, Boonstra A, Groothoff JW, De Vries J, Goeken LN, Eisma WH. The timed "up and go" test: Reliability and validity in persons with unilateral lower limb amputation. Arch Phys Med Rehabil. 1999;80(7):825-28. [PMID: 10414769]

20. Field AP. Discovering statistics using SPSS for Windows: Advanced techniques for the beginner. London (England): Sage Publications; 2000. p. 96-101.

Submitted for publication May 22, 2006. Accepted in revised form May 11, 2007. 